\title{
Multiparameter fractional Brownian motion and quasi-linear stochastic partial differential equations
}

\author{
Bernt $\varnothing \mathrm{ksendal}^{1,2} \quad$ Tusheng Zhang ${ }^{3,4}$
}

February 16, 2000

\begin{abstract}
We develop a multiparameter white noise theory for fractional Brownian motion with Hurst multiparameter $H=\left(H_{1}, \ldots, H_{d}\right) \in\left(\frac{1}{2}, 1\right)^{d}$. The theory is used to solve the linear and a quasi-linear heat equation driven by multiparameter fractional white noise. It is proved that for some values of $H$ (depending on the dimension) the solution has a jointly continuous version in $t$ and $x$.
\end{abstract}

\section{Introduction}

Recall that if $0<H<1$ then the (1-parameter) fractional Brownian motion with Hurst parameter $H$ is the Gaussian process $B_{H}(t)=B_{H}(t, \omega) ; t \in \mathbf{R}, \omega \in \Omega$ satisfying

$$
B_{H}(0)=E\left[B_{H}(t)\right]=0 \quad \text { for all } t \in \mathbf{R}
$$

and

$$
E\left[B_{H}(s) B_{H}(t)\right]=\frac{1}{2}\left\{|s|^{2 H}+|t|^{2 H}-|s-t|^{2 H}\right\} \quad \text { for all } s, t \in \mathbf{R} .
$$

Here $E$ denotes the expectation with respect to the probability law $P$ for $\left\{B_{H}(t, \omega)\right\}_{t \in \mathbf{R}, \omega \in \Omega}$, where $(\Omega, \mathcal{F})$ is a measurable space.

If $H=\frac{1}{2}$ then $B_{H}(t)$ coincides with the standard Brownian motion $B(t)$. Much of the recent interest in fractional Brownian motion stems from its property that if $H>\frac{1}{2}$ then $B_{H}(t)$ has a long range dependence, in the sense that

$$
\sum_{n=1}^{\infty} E\left[B_{H}(1)\left(B_{H}(n+1)-B_{H}(n)\right)\right]=\infty .
$$

\footnotetext{
1 Dept. of Mathematics, University of Oslo, P. O. Box 1053 Blindern, N-0316 Oslo, Norway. email: oksendal@math.uio.no

${ }^{2}$ Norwegian School of Economics and Business Administration, Helleveien 30, N-5045 Bergen, Norway.

${ }^{3}$ Dept. of Mathematics, Agder College, N-4684 Kristiansand, Norway.

${ }^{4}$ Dept. of Mathematics, Cornell University, Ithaca, NY 14853-4201, USA.

email: tusheng@math.cornell.edu
} 
Moreover, for any $H \in(0,1)$ and $\alpha>0$ the law of $\left\{B_{H}(\alpha t)\right\}_{t \in \mathbf{R}}$ is the same as the law of $\left\{\alpha^{H} B_{H}(t)\right\}_{t \in \mathbf{R}}$, i.e. $B_{H}(t)$ is $H$-self-similar.

For more information on 1-parameter fractional Brownian motion see e.g. [MV], [NVV] and the references therein.

Recently a stochastic calculus based on Itô-type of integration with respect to $B_{H}(t)$ has been constructed for $H>\frac{1}{2}$ [DHP]. Subsequently a corresponding fractional white noise theory has been developed [HØ], and this has been used to study the corresponding fractional models in mathematical finance [HØ], [HØS].

As in [H1], [H2] and [HØZ] we define d-parameter fractional Brownian motion $B_{H}(x)$; $x=\left(x_{1}, \ldots, x_{d}\right) \in \mathbf{R}^{d}$ with Hurst parameter $H=\left(H_{1}, \ldots, H_{d}\right) \in(0,1)^{d}$ as a Gaussian process on $\mathbf{R}^{d}$ with mean

$$
E\left[B_{H}(x)\right]=0 \quad \text { for all } x \in \mathbf{R}^{d}
$$

and covariance

$$
E\left[B_{H}(x) B_{H}(y)\right]=\left(\frac{1}{2}\right)^{d} \prod_{i=1}^{d}\left(\left|x_{i}\right|^{2 H_{i}}+\left|y_{i}\right|^{2 H_{i}}-\left|x_{i}-y_{i}\right|^{2 H_{i}}\right)
$$

We also assume that

$$
B_{H}(0)=0 \quad \text { a.s. }
$$

From now on we will assume that

$$
\frac{1}{2}<H_{i}<1 \quad \text { for } i=1, \ldots, d .
$$

The purpose of this paper is to extend the fractional white noise theory to the multiparameter case and use this theory to study the linear and quasilinear heat equation with a fractional white noise force.

\section{Multiparameter fractional white noise}

In this section we outline how the multiparameter white noise theory for standard Brownian motion (see e.g. [HKPS], [HØUZ] or [K]) can be extended to fractional Brownian motion. In the 1-parameter case such an extension was presented in [HØ]. The following outline will follow the introduction in [HØZ] closely.

Fix a parameter dimension $d \in \mathbf{N}$ and a Hurst parameter

$$
H=\left(H_{1}, \ldots, H_{d}\right) \in\left(\frac{1}{2}, 1\right)^{d} .
$$

Define

$$
\varphi(x, y)=\varphi_{H}(x, y)=\prod_{i=1}^{d} H_{i}\left(2 H_{i}-1\right)\left|x_{i}-y_{i}\right|^{2 H_{i}-2}
$$


for $x=\left(x_{1}, \ldots, x_{d}\right) \in \mathbf{R}^{d}, y=\left(y_{1}, \ldots, y_{d}\right) \in \mathbf{R}^{d}$.

Let $L_{\varphi}^{2}\left(\mathbf{R}^{d}\right)$ be the space of measurable functions $f: \mathbf{R}^{d} \rightarrow \mathbf{R}$ satisfying

$$
|f|_{\varphi}^{2}:=\int_{\mathbf{R}^{d}} \int_{\mathbf{R}^{d}} f(x) f(y) \varphi(x, y) d x d y<\infty
$$

where $d x=d x_{1} \ldots d x_{d}$ and $d y=d y_{1} \ldots d y_{d}$ denotes Lebesgue measure.

Then $L_{\varphi}^{2}\left(\mathbf{R}^{d}\right)$ is a separable Hilbert space with the inner product

$$
(f, g)_{\varphi}=\int_{\mathbf{R}^{d}} \int_{\mathbf{R}^{d}} f(x) g(y) \varphi(x, y) d x d y ; \quad f, g \in L_{\varphi}^{2}\left(\mathbf{R}^{d}\right) .
$$

In fact, we have (see [HØ, Lemma 2.1] for the case $d=1$ ):

Lemma 2.1 For $f \in L_{\varphi}^{2}\left(\mathbf{R}^{d}\right)$ and $u=\left(u_{1}, \ldots, u_{d}\right) \in \mathbf{R}^{d}$ define

$$
\Gamma_{\varphi} f(u)=\int_{u_{1}}^{\infty} \ldots \int_{u_{d}}^{\infty} f\left(x_{1}, \ldots, x_{d}\right) \prod_{i=1}^{d} c_{H_{i}}\left(x_{i}-u_{i}\right)^{H_{i}-3 / 2} d x_{1} \ldots d x_{d},
$$

where

$$
c_{H_{i}}=\sqrt{\frac{H_{i}\left(2 H_{i}-1\right) \cdot \Gamma\left(\frac{3}{2}-H_{i}\right)}{\Gamma\left(H_{i}-\frac{1}{2}\right) \cdot \Gamma\left(2-2 H_{i}\right)}} ; \quad i=1, \ldots, d .
$$

Then $\Gamma_{\varphi}$ is an isometry from $L_{\varphi}^{2}\left(\mathbf{R}^{d}\right)$ into $L^{2}\left(\mathbf{R}^{d}\right)$.

Proof. For $f, g \in L_{\varphi}^{2}\left(\mathbf{R}^{d}\right)$ we have

$$
\begin{aligned}
& \left(\Gamma_{\varphi}(f), \Gamma_{\varphi}(g)\right)_{L^{2}\left(\mathbf{R}^{d}\right)} \\
& =\int_{\mathbf{R}^{d}}\left(\int_{u_{i}}^{\infty} \ldots \int_{u_{d}}^{\infty} f(x) \prod_{i=1}^{d} c_{H_{i}}\left(x_{i}-u_{i}\right)^{H_{i}-3 / 2} d x\right) \\
& \quad \cdot\left(\int_{u_{1}}^{\infty} \ldots \int_{u_{d}}^{\infty} g(y) \prod_{i=1}^{d} c_{H_{i}}\left(y_{i}-u_{i}\right)^{H_{i}-3 / 2} d y\right) d u_{1} \ldots d u_{d} \\
& =\int_{\mathbf{R}^{d}} \int_{\mathbf{R}^{d}} f(x) g(y)\left(\prod_{i=1}^{d} \int_{-\infty}^{x_{i} \wedge y_{i}} c_{H_{i}}^{2}\left(x_{i}-u_{i}\right)^{H_{i}-3 / 2}\left(y_{i}-u_{i}\right)^{H_{i}-3 / 2} d u_{i}\right) d x d y \\
& =\int_{\mathbf{R}^{d}} \int_{\mathbf{R}^{d}} f(x) g(y) \varphi(x, y) d x d y,
\end{aligned}
$$


where we have used the fact that (see e.g. [GN, p. 404])

$$
\int_{-\infty}^{x_{i} \wedge y_{i}} c_{H_{i}}^{2}\left(x_{i}-u_{i}\right)^{H_{i}-3 / 2}\left(y_{i}-u_{i}\right)^{H_{i}-3 / 2} d u_{i}=H_{i}\left(2 H_{i}-1\right)\left|x_{i}-y_{i}\right|^{2 H_{i}-2} .
$$

Let $\mathcal{S}\left(\mathbf{R}^{d}\right)$ be the Schwartz space of rapidly decreasing smooth functions on $\mathbf{R}^{d}$. The dual of $\mathcal{S}\left(\mathbf{R}^{d}\right)$, the space of tempered distributions, is denoted by $\mathcal{S}^{\prime}\left(\mathbf{R}^{d}\right)$. The functional

$$
f \rightarrow \exp \left(-\frac{1}{2}|f|_{\varphi}^{2}\right) ; \quad f \in \mathcal{S}\left(\mathbf{R}^{d}\right)
$$

is positive definite on $\mathcal{S}\left(\mathbf{R}^{d}\right)$, so by the Bochner-Minlos theorem there exists a probability measure $\mu_{\varphi}$ on $\mathcal{S}^{\prime}\left(\mathbf{R}^{d}\right)$ such that

$$
\int_{\mathcal{S}^{\prime}\left(\mathbf{R}^{d}\right)} e^{i\langle\omega, f\rangle} d \mu_{\varphi}(\omega)=e^{-\frac{1}{2}|f|_{\varphi}^{2}} ; \quad f \in \mathcal{S}\left(\mathbf{R}^{d}\right)
$$

where $\langle\omega, f\rangle$ denotes the action of $\omega \in \Omega:=\mathcal{S}^{\prime}\left(\mathbf{R}^{d}\right)$ on $f \in \mathcal{S}\left(\mathbf{R}^{d}\right)$. From (2.8) one can deduce that if $f_{n} \in \mathcal{S}\left(\mathbf{R}^{d}\right)$ and $f_{n} \rightarrow f$ in $L_{\varphi}^{2}\left(\mathbf{R}^{d}\right)$ then

$$
\langle\omega, f\rangle:=\lim _{n \rightarrow \infty}\left\langle\omega, f_{n}\right\rangle \quad \text { exists in } L^{2}\left(\mu_{\varphi}\right)
$$

and defines a Gaussian random variable. Moreover,

$$
E[\langle\cdot, f\rangle]=0
$$

and

$$
E[\langle\cdot, f\rangle\langle\cdot, g\rangle]=(f, g)_{\varphi} \quad \text { for } f, g \in L_{\varphi}^{2}\left(\mathbf{R}^{d}\right) .
$$

Here, and in the following, $E[\cdot]=E_{\mu_{\varphi}}[\cdot]$ denotes the expectation with respect to $\mu_{\varphi}$.

In particular, we may define

$$
\widetilde{B}_{H}(x)=\left\langle\omega, \mathcal{X}_{[0, x]}(\cdot)\right\rangle ; \quad x=\left(x_{1}, \ldots, x_{d}\right) \in \mathbf{R}^{d}
$$

where

$$
\mathcal{X}_{[0, x]}(y)=\prod_{i=1}^{d} \mathcal{X}_{\left[0, x_{i}\right]}\left(y_{i}\right) \quad \text { for } \quad y=\left(y_{1}, \ldots, y_{d}\right) \in \mathbf{R}^{d}
$$

and

$$
\mathcal{X}_{\left[0, x_{i}\right]}\left(y_{i}\right)= \begin{cases}1 & \text { if } 0 \leq y_{i} \leq x_{i} \\ -1 & \text { if } x_{i} \leq y_{i} \leq 0, \text { except } x_{i}=y_{i}=0 \\ 0 & \text { otherwise }\end{cases}
$$


Using (2.10)-(2.11) and Kolmogorov's criterion, we see that $\widetilde{B}_{H}(x) ; x \in \mathbf{R}^{d}$ is a Gaussian process and it has a continuous version. Furthermore, we see that

$$
E\left[B_{H}(x)\right]=0
$$

and

$$
E\left[B_{H}(x) B_{H}(y)\right]=\left(\frac{1}{2}\right)^{d} \prod_{i=1}^{d}\left(\left|x_{i}\right|^{2 H_{i}}+|y|^{2 H_{i}}-\left|x_{i}-y_{i}\right|^{2 H_{i}}\right) .
$$

Therefore $B_{H}(x) ; x \in \mathbf{R}^{d}$ is a $d$-parameter fractional Brownian motion with Hurst parameter $H=\left(H_{1}, \ldots, H_{d}\right) \in\left(\frac{1}{2}, 1\right)^{d}$ (see (1.3)-(1.5)). It is this version of $B_{H}(x)$ we will use from now on.

Let $f \in L_{\varphi}^{2}\left(\mathbf{R}^{d}\right)$. The stochastic integral of $f$ with respect to the fractional Brownian motion $B_{H}(x)$ is the Gaussian random variable on $\Omega$ defined by

$$
\int_{\mathbf{R}^{d}} f(x) d B_{H}(x)=\int_{\mathbf{R}^{d}} f(x) d B_{H}(x, \omega)=\langle\omega, f\rangle .
$$

Note that this is a natural definition from the point of view of Riemann sums:

If $f_{n}$ is a simple integrand of the form

$$
f_{n}(x)=\sum_{j=1}^{N_{n}} a_{j}^{(n)} \mathcal{X}_{\left(-\infty, y_{j}\right)}(x)
$$

then (2.13) gives

$$
\int_{\mathbf{R}^{d}} f_{n}(x) d B_{H}(x)=\left\langle\omega, f_{n}\right\rangle=\sum_{j=1}^{N_{n}} a_{j}^{(n)} B_{H}\left(y_{j}\right)
$$

and if $f_{n} \rightarrow f$ in $L_{\varphi}^{2}\left(\mathbf{R}^{d}\right)$ then by (2.9) we have, as desired, that

$$
\int_{\mathbf{R}^{d}} f_{n}(x) d B_{H}(x)=\left\langle\omega, f_{n}\right\rangle \rightarrow\langle\omega, f\rangle=\int_{\mathbf{R}^{d}} f(x) d B_{H}(x) .
$$

Note that from (2.14) and (2.11) we have the fractional Ito isometry

$$
E\left[\left(\int_{\mathbf{R}^{d}} f(x) d B_{H}(x)\right)^{2}\right]=|f|_{\varphi}^{2} \quad \text { for } f \in L_{\varphi}^{2}\left(\mathbf{R}^{d}\right) .
$$

As in [HØZ] we now proceed in analogy with [HØUZ] (as done in [HØ] in the 1parameter case) to obtain a multiparameter fractional chaos expansion: 
Let

$$
h_{n}(t)=(-1)^{n} e^{t^{2} / 2} \frac{d^{n}}{d t^{n}}\left(e^{-t^{2} / 2}\right) ; \quad t \in \mathbf{R}, \quad n=0,1,2, \ldots
$$

be the standard Hermite polynomials and let

$$
\widetilde{h}_{n}(t)=\pi^{-1 / 4}((n-1) !)^{-1 / 2} h_{n-1}(\sqrt{2} t) e^{-t^{2} / 2} ; \quad n=1,2, \ldots
$$

be the Hermite functions. Let $\mathbf{N}=\{1,2, \ldots\}$. For $\alpha \in \mathbf{N}^{d}$ let $\eta_{\alpha}(x)=\prod_{i=1}^{d} \widetilde{h}_{\alpha_{i}}\left(x_{i}\right)$. Then $\left\{\eta_{\alpha}\right\}_{\alpha \in \mathbf{N}^{d}}$ constitutes an orthonormal basis of $L^{2}\left(\mathbf{R}^{d}\right)$. Therefore

$$
e_{\alpha}(x):=\Gamma_{\varphi}^{-1}\left(\eta_{\alpha}\right)(x) ; \quad \alpha \in \mathbf{N}^{d}, \quad x \in \mathbf{R}^{d}
$$

constitutes an orthonormal basis of $L_{\varphi}^{2}\left(\mathbf{R}^{d}\right)$. From now on we let $\left\{\alpha^{(i)}\right\}_{i=1}^{\infty}$ be a fixed ordering of $\mathbf{N}^{d}$ with the property that

$$
i<j \Rightarrow\left|\alpha^{(i)}\right| \leq\left|\alpha^{(j)}\right|
$$

and we write

$$
e_{n}(x):=e_{\alpha^{(n)}}(x) . \quad(\text { See }(2.2 .7) \text { in }[\mathrm{H} \varnothing \mathrm{UZ}])
$$

Then just as in [HØ, Lemma 3.1] we can prove

Lemma 2.2 There exists a locally bounded function $C(x)$ on $\mathbf{R}^{d}$ such that

$$
\left|\int_{\mathbf{R}^{d}} e_{n}(y) \varphi(x, y) d y\right| \leq C(x) \prod_{i=1}^{d}\left(\alpha_{i}^{(n)}\right)^{1 / 6}
$$

Let $\mathcal{J}=\left(\mathbf{N}_{0}^{\mathbf{N}}\right)_{c}$ denote the set of all (finite) multi-indices $\alpha=\left(\alpha_{1}, \ldots, \alpha_{m}\right)$ with $\alpha_{i} \in \mathbf{N}_{0}:=\mathbf{N} \cup\{0\}$. Then if $\alpha=\left(\alpha_{1}, \ldots, \alpha_{m}\right) \in \mathcal{J}$ we define

$$
\mathcal{H}_{\alpha}(\omega)=h_{\alpha_{1}}\left(\left\langle\omega, e_{1}\right\rangle\right) \cdots h_{\alpha_{m}}\left(\left\langle\omega, e_{m}\right\rangle\right) .
$$

In particular, if we put

$$
\varepsilon^{(i)}=(0,0, \ldots, 1) \quad(\text { the } i \text { 'th unit vector })
$$

then by (2.14) we get

$$
\mathcal{H}_{\varepsilon^{(i)}}(\omega)=h_{1}\left(\left\langle\omega, e_{i}\right\rangle\right)=\left\langle\omega, e_{i}\right\rangle=\int_{\mathbf{R}^{d}} e_{i}(x) d B_{H}(x) .
$$

As is well-known in a more general context (see e.g. [J, Theorem 2.6]) we have the following Wiener-Itô chaos expansion theorem (see also [DHP] and [HØ]): 
Theorem 2.3 Let $F \in L^{2}\left(\mu_{\varphi}\right)$. Then there exist constants $c_{\alpha} \in \mathbf{R}$ for $\alpha \in \mathcal{J}$, such that

$$
\left.F(\omega)=\sum_{\alpha \in \mathcal{J}} c_{\alpha} \mathcal{H}_{\alpha}(\omega) \quad \text { (convergence in } L^{2}\left(\mu_{\varphi}\right)\right)
$$

Moreover, we have the isometry,

$$
\|F\|_{L^{2}\left(\mu_{\varphi}\right)}^{2}=\sum_{\alpha \in \mathcal{J}} \alpha ! c_{\alpha}^{2}
$$

where $\alpha !=\alpha_{1} ! \alpha_{2} ! \ldots \alpha_{m} !$ if $\alpha=\left(\alpha_{1}, \ldots, \alpha_{m}\right) \in \mathcal{J}$.

Example 2.4 If $F(\omega)=\langle\omega, f\rangle$ for some $f \in L_{\varphi}^{2}\left(\mathbf{R}^{d}\right)$, then $F$ has the expansion

$$
F(\omega)=\left\langle\omega, \sum_{i=1}^{\infty}\left(f, e_{i}\right)_{\varphi} e_{i}\right\rangle=\sum_{i=1}^{\infty}\left(f, e_{i}\right)_{\varphi} \mathcal{H}_{\varepsilon^{(i)}}(\omega) .
$$

In particular, for d-parameter fractional Brownian motion we get, by (2.12),

$$
\begin{aligned}
B_{H}(x) & =\left\langle\omega, \mathcal{X}_{[0, x]}(\cdot)\right\rangle=\sum_{i=1}^{\infty}\left(\mathcal{X}_{[0, x]}, e_{i}\right)_{\varphi} \mathcal{H}_{\varepsilon^{(i)}}(\omega) \\
& =\sum_{i=1}^{\infty}\left[\int_{0}^{x}\left(\int_{\mathbf{R}^{d}} e_{i}(v) \varphi(u, v) d v\right) d u\right] \mathcal{H}_{\varepsilon^{(i)}}(\omega)
\end{aligned}
$$

where $\int_{0}^{x}=\int_{0}^{x_{d}} \ldots \int_{0}^{x_{1}}$ and $\int_{0}^{x_{i}}=-\int_{x_{i}}^{0}$ if $x_{i}<0$.

Next we proceed as in [HØUZ] to define the multiparameter fractional Hida test function space $(\mathcal{S})_{H}$ and distribution space $(\mathcal{S})_{H}^{*}$ :

Definition 2.5 a) (The multiparameter fractional Hida test function spaces) For $k \in \mathbf{N}$ define $(\mathcal{S})_{H, k}$ to be the space of all

$$
\psi(\omega)=\sum_{\alpha \in \mathcal{J}} a_{\alpha} \mathcal{H}_{\alpha}(\omega) \in L^{2}\left(\mu_{\varphi}\right)
$$

such that

$$
\|\psi\|_{H, k}^{2}:=\sum_{\alpha \in \mathcal{J}} \alpha ! a_{\alpha}^{2}(2 \mathbf{N})^{k \alpha}<\infty
$$

where

$$
(2 \mathbf{N})^{\gamma}=\prod_{j}(2 j)^{\gamma_{j}} \quad \text { if } \quad \gamma=\left(\gamma_{1}, \ldots, \gamma_{m}\right) \in \mathcal{J}
$$


Define $(\mathcal{S})_{H}=\bigcap_{k=1}^{\infty}(\mathcal{S})_{H, k}$ with the projective topology.

b) (The multiparameter fractional Hida distribution spaces)

For $q \in \mathbf{N}$ let $(\mathcal{S})_{H,-q}^{*}$ be the space of all formal expansions

$$
G(\omega)=\sum_{\beta \in \mathcal{J}} b_{\beta} \mathcal{H}_{\alpha}(\omega)
$$

such that

$$
\|G\|_{H,-q}^{2}:=\sum_{\beta \in \mathcal{J}} \beta ! b_{\beta}^{2}(2 \mathbf{N})^{-q \beta}<\infty
$$

Define

$$
(\mathcal{S})_{H}^{*}=\bigcup_{q=1}^{\infty}(\mathcal{S})_{H,-q}^{*}
$$

with the inductive topology. Then $(\mathcal{S})_{H}^{*}$ becomes the dual of $(\mathcal{S})_{H}$ when the action of $G \in(\mathcal{S})_{H}^{*}$ given by (2.26) on $\psi \in(\mathcal{S})_{H}$ given by (2.24) is defined by

$$
\langle\langle G, \psi\rangle\rangle=\sum_{\alpha \in \mathcal{J}} \alpha ! a_{\alpha} b_{\alpha}
$$

\section{Example 2.6 (Multiparameter fractional white noise)}

Define, for $y \in \mathbf{R}^{d}$

$$
W_{H}(y)=\sum_{i=1}^{\infty}\left[\int_{\mathbf{R}^{d}} e_{i}(v) \varphi(y, v) d v\right] \mathcal{H}_{\varepsilon^{(i)}}(\omega)
$$

Then as in [HØ, Example 3.6] we obtain that $W_{H}(y) \in(\mathcal{S})_{H}^{*}$ for all $y$. Moreover, $W_{H}(y)$ is integrable in $(\mathcal{S})_{H}^{*}$ for $0 \leq y_{i} \leq x_{i} ; i=1, \ldots, d$, and

$$
\int_{0}^{x} W_{H}(y) d y=\sum_{i=1}^{\infty}\left[\int_{0}^{x}\left(\int_{\mathbf{R}^{d}} e_{i}(v) \varphi(y, v) d v\right) d y\right] \mathcal{H}_{\varepsilon^{(i)}}(\omega)=B_{H}(x)
$$

by $(2.23)$. Therefore $B_{H}(x)$ is differentiable with respect to $x$ in $(\mathcal{S})_{H}^{*}$ and we have

$$
\frac{\partial^{d}}{\partial x_{1} \ldots \partial x_{d}} B_{H}(x)=W_{H}(x) \quad \text { in } \quad(\mathcal{S})_{H}^{*} .
$$

This justifies the name (multiparameter) fractional white noise for $W_{H}(x)$.

The Wick product is defined just as in [HØUZ] and [HØ]: 
Definition 2.7 Suppose $F(\omega)=\sum_{\alpha \in \mathcal{J}} a_{\alpha} \mathcal{H}_{\alpha}(\omega)$ and $G(\omega)=\sum_{\beta \in \mathcal{J}} b_{\beta} \mathcal{H}_{\beta}(\omega)$ both belong to $(\mathcal{S})_{H}^{*}$. Then we define their Wick product $(F \diamond G)(\omega)$ by

$$
(F \diamond G)(\omega)=\sum_{\alpha, \beta \in \mathcal{J}} a_{\alpha} b_{\beta} \mathcal{H}_{\alpha+\beta}(\omega)=\sum_{\gamma \in \mathcal{J}}\left(\sum_{\alpha+\beta=\gamma} a_{\alpha} b_{\beta}\right) \mathcal{H}_{\gamma}(\omega) .
$$

Example 2.8 a) ([HØ, Example 3.9]) If $f, g \in L_{\varphi}^{2}\left(\mathbf{R}^{d}\right)$ then

$$
\left(\int_{\mathbf{R}^{d}} f d B_{H}\right) \diamond\left(\int_{\mathbf{R}^{d}} g d B_{H}\right)=\left(\int_{\mathbf{R}^{d}} f d B_{H}\right) \cdot\left(\int_{\mathbf{R}^{d}} g d B_{H}\right)-(f, g)_{\varphi} .
$$

b) ([HØ, Example 3.10]) If $f \in L_{\varphi}^{2}\left(\mathbf{R}^{d}\right)$ then

$$
\exp ^{\diamond}(\langle\omega, f\rangle):=\sum_{n=1}^{\infty} \frac{1}{n !}\langle\omega, f\rangle^{\diamond n}
$$

converges in $(\mathcal{S})_{H}^{*}$ and is given by

$$
\exp ^{\diamond}(\langle\omega, f\rangle)=\exp \left(\langle\omega, f\rangle-\frac{1}{2}|f|_{\varphi}^{2}\right) .
$$

We now use multiparameter fractional white noise to define integration with respect to multiparameter fractional Brownian motion, just as in [HØ, Definition 3.11] for the 1-parameter case:

Definition 2.9 Suppose $Y: \mathbf{R}^{d} \rightarrow(\mathcal{S})_{H}^{*}$ is a given function such that $Y(x) \diamond W_{H}(x)$ is integrable in $(\mathcal{S})_{H}^{*}$ for $x \in \mathbf{R}^{d}$. Then we define the multiparameter fractional stochastic integral (of Itô type) of $Y(x)$ by

$$
\int_{\mathbf{R}^{d}} Y(x) d B_{H}(x)=\int_{\mathbf{R}^{d}} Y(x) \diamond W_{H}(x) d x .
$$

Remark 2.10 If $H=\frac{1}{2}$ this definition gives an extension of the Itô-Skorohod integral. See [HØUZ, Section 2.5] for more details.

\section{The linear heat equation driven by fractional white noise}

In this section we illustrate the theory above by applying it to the linear stochastic fractional heat equation

$$
\begin{aligned}
& \frac{\partial U}{\partial t}(t, x)=\frac{1}{2} \Delta U(t, x)+W_{H}(t, x) ; \quad t \in(0, \infty), \quad x \in D \subset \mathbf{R}^{n} \\
& U(0, x)=0 ; \quad x \in D \\
& U(t, x)=0 ; \quad t \geq 0, \quad x \in \partial D
\end{aligned}
$$


Here $W_{H}(t, x)$ is the fractional white noise with Hurst parameter $H=\left(H_{0}, H_{1}, \ldots, H_{n}\right) \in$ $\left(\frac{1}{2}, 1\right)^{n+1}, \Delta=\sum_{i=1}^{n} \frac{\partial^{2}}{\partial x_{i}^{2}}$ is the Laplace operator, $D \subset \mathbf{R}^{n}$ is a bounded open set with smooth boundary $\partial D, 0 \leq T \leq \infty$ is a constant. We are looking for a solution $U:[0, \infty) \times \bar{D} \rightarrow$ $(\mathcal{S})_{H}^{*}$ which is continuously differentiable in $(t, x)$ and twice continuously differentiable in $x$, i.e. belongs to $C^{1,2}\left((0, \infty) \times D ;(\mathcal{S})_{H}^{*}\right)$, and which satisfies (3.1) in the strong sense (as an $(\mathcal{S})_{H^{*}}^{*}$-valued function).

Based on the corresponding solution in the deterministic case (with $W_{H}(t, x)$ replaced by a bounded deterministic function) it is natural to guess that the solution will be

$$
U(t, x)=\int_{0}^{t} \int_{D} W_{H}(s, y) G_{t-s}(x, y) d y d s
$$

where $G_{t-s}(x, y)$ is the Green function for the heat operator $\frac{\partial}{\partial t}-\frac{1}{2} \Delta$. It is well-known [D] that $G$ is smooth in $(0, T) \times D$ and that

$$
G_{u}(x, y) \sim u^{-n / 2} \exp \left(-\frac{|x-y|^{2}}{\delta u}\right) \quad \text { in }(0, \infty) \times D,
$$

where the notation $X \sim Y$ means that

$$
\frac{1}{C} X \leq Y \leq C X \quad \text { in }(0, \infty) \times D,
$$

for some positive constant $C<\infty$ depending only on $D$.

We use this to verify that $U(t, x) \in \mathcal{S}_{H}^{*}$ for all $(t, x) \in[0, \infty) \times \bar{D}$ :

Using (2.29) we see that the expansion of $U(t, x)$ is

$$
\begin{aligned}
U(t, x) & =\int_{0}^{t} \int_{D} G_{t-s}(x, y) \sum_{k=1}^{\infty}\left[\int_{\mathbf{R}^{n}} e_{k}(v) \varphi(y, v) d v\right] \mathcal{H}_{\varepsilon^{(k)}}(\omega) d y d s \\
& =\sum_{k=1}^{\infty} b_{k}(t, x) \mathcal{H}_{\varepsilon^{(k)}}(\omega)
\end{aligned}
$$

where

$$
b_{k}(t, x)=b_{\varepsilon^{(k)}}(t, x)=\int_{0}^{t} \int_{D} G_{t-s}(x, y)\left[\int_{\mathbf{R}^{n}} e_{k}(v) \varphi(y, v) d v\right] d y d s
$$

In the following $C$ denote constants, not necessarily the same from place to place. From 
Lemma 2.2 and (3.7) we obtain that

$$
\begin{aligned}
& \left|b_{k}(t, x)\right| \leq C \prod_{i=1}^{d}\left(\alpha_{i}^{(k)}\right)^{1 / 6} \int_{0}^{t} \int_{D} G_{t-s}(x, y) d y d s \\
& \leq C \prod_{i=1}^{d}\left(\alpha_{i}^{(k)}\right)^{1 / 6} \int_{0}^{t}\left(\int_{\mathbf{R}^{n}} s^{-n / 2} \exp \left(-\frac{y^{2}}{\delta s}\right) d y\right) d s \\
& \quad \leq \sqrt{\delta s} z \\
& \quad \leq \prod_{i=1}^{d}\left(\alpha_{i}^{(k)}\right)^{1 / 6} \int_{0}^{t}\left(\int_{\mathbf{R}^{n}} s^{-n / 2} \exp \left(-z^{2}\right)(\delta s)^{n / 2} d z\right) d s \\
& \quad=C \prod_{i=1}^{d}\left(\alpha_{i}^{(k)}\right)^{1 / 6} t .
\end{aligned}
$$

Therefore

$$
\begin{aligned}
& \sum_{k=1}^{\infty} b_{k}^{2}(t, x)(2 \mathbf{N})^{-q \varepsilon^{(k)}} \\
& \quad \leq C(t) \sum_{k=1}^{\infty} \prod_{i=1}^{d}\left(\alpha_{i}^{(k)}\right)^{1 / 3}(2 k)^{-q} \leq \sum_{k=1}^{\infty} k^{d / 3}(2 k)^{-q}<\infty \quad \text { for } \quad q>\frac{d+3}{3} .
\end{aligned}
$$

Here we used the fact $\left|\alpha^{(k)}\right| \leq k$, which is the consequence of the special order. Hence $U(t, x) \in(\mathcal{S})_{H,-q}^{*}$ for all $q>\frac{d+3}{3}$, for all $t, x$.

In fact, this estimate also shows that $U(t, x)$ is uniformly continuous as a function from $[0, T] \times \bar{D}$ into $(\mathcal{S})_{H}^{*}$ for any $T<\infty$ and that $U(t, x)$ satisfies (3.2) and (3.3). Moreover, by the properties of $G_{t-s}(x, y)$ we get from (3.4) that

$$
\begin{gathered}
\frac{\partial U}{\partial t}(t, x)-\Delta U(t, x)=\int_{0}^{t} \int_{D} W_{H}(s, y)\left(\frac{\partial}{\partial t}-\Delta\right) G_{t-s}(x, y) d y d s+W_{H}(t, x) \\
=W_{H}(t, x), \quad \text { so } U(t, x) \text { satisfies (3.1) also . }
\end{gathered}
$$

In the standard white noise case $\left(H_{i}=\frac{1}{2}\right.$ for all $\left.i\right)$ the same solution formula (3.4) holds. In this case we see that the solution $U(t, x)$ belongs to $L^{2}(\mu)$ ( $\mu$ being the standard white noise measure) iff

$$
E_{\mu}\left[U^{2}(t, x)\right]=\int_{0}^{t} \int_{D} G_{t-s}^{2}(x, y) d y d s<\infty
$$


Now, if $D \subset\left(-\frac{1}{2} R, \frac{1}{2} R\right)^{n}$ and we put $F=[-R, R]^{n}$,

$$
\begin{aligned}
\int_{0}^{t} \int_{D} G_{t-s}^{2}(x, y) d s d y & \sim \int_{0}^{t} \int_{D} s^{-n} \exp \left(-\frac{2 y^{2}}{\delta s}\right) d y d s \\
& \sim \int_{0}^{t}\left(\int_{F / \sqrt{s}} s^{-n / 2} \exp \left(-\frac{2 z^{2}}{\delta}\right) d z\right) d s .
\end{aligned}
$$

Hence

$$
E_{\mu}\left[U^{2}(t, x)\right]<\infty \Longleftrightarrow n=1
$$

Next, consider the fractional case $\frac{1}{2}<H_{i}<1$ for all $i$. Then

$$
\begin{gathered}
E_{\mu_{\varphi}}\left[U^{2}(t, x)\right]=\int_{0}^{t} \int_{0}^{t} \int_{D} \int_{D} G_{t-r}(x, y) G_{t-s}(x, z) \varphi(r, s, y, z) d r d s d y d z \\
\sim \int_{0}^{t} \int_{0}^{t} \int_{D} \int_{D} r^{-n / 2} s^{-n / 2} \exp \left(-\frac{|x-y|^{2}}{\delta r}\right) \exp \left(-\frac{|x-z|^{2}}{\delta s}\right) \\
\cdot|r-s|^{2 H_{0}-2} \prod_{i=1}^{n}\left|y_{i}-z_{i}\right|^{2 H_{i}-2} d y_{1} \ldots d y_{n} d z_{1} \ldots d z_{n} d r d s .
\end{gathered}
$$

Choose $1<q<p<\infty$ such that $\frac{1}{p}+\frac{1}{q}=1$. By the Hölder inequality we have

$$
\begin{aligned}
\prod_{i=1}^{n} \int_{\frac{1}{2} R}^{\frac{1}{2} R} & \int_{\frac{1}{2} R}^{\frac{1}{2} R} \exp \left(-\frac{\left|x_{i}-y_{i}\right|^{2}}{\delta r}-\frac{\left|x_{i}-z_{i}\right|^{2}}{\delta s}\right)\left|y_{i}-z_{i}\right|^{2 H_{i}-2} d y_{i} d z_{i} \\
\leq & \prod_{i=1}^{n} \int_{\frac{1}{2} R}^{\frac{1}{2} R} \exp \left(-\frac{\left|x_{i}-y_{i}\right|^{2}}{\delta r}\right) d y_{i}\left\{\left[\int_{\frac{1}{2} R}^{\frac{1}{2} R} \exp \left(-\frac{p\left|x_{i}-z_{i}\right|^{2}}{\delta s}\right) d z_{i}\right]^{1 / p}\right. \\
\left.\cdot\left[\int_{\frac{1}{2} R}^{\frac{1}{2} R}\left|y_{i}-z_{i}\right|^{q\left(2 H_{i}-2\right)} d z_{i}\right]^{1 / q}\right\} & \text { if } q\left(2 H_{i}-2\right)>-1 .
\end{aligned}
$$

Substituted into (3.13) this gives

$$
\begin{gathered}
E_{\mu_{\varphi}}\left[U^{2}(t, x)\right] \leq C(p) \int_{0}^{t} \int_{0}^{t}(s)^{-\frac{n}{2}\left(1-\frac{1}{p}\right)}|r-s|^{2 H_{0}-2} d r d s \\
<\infty \quad \text { if } \quad n<\frac{2 p}{p-1} .
\end{gathered}
$$


Combined with the requirement $q\left(2 H_{i}-2\right)>-1$ we obtain from this that

$$
E_{\mu_{\varphi}}\left[U^{2}(t, x)\right]<\infty \quad \text { if } n<\frac{1}{1-H_{i}} \text { for } 1 \leq i \leq n
$$

We summarize what we have proved:

Theorem 3.1 a) For any space dimension $n$ there is a unique strong solution $U(t, x)$ : $[0, \infty) \times D \rightarrow(\mathcal{S})_{H}^{*}$ of the fractional heat equation (3.1)-(3.3). The solution is given by

$$
U(t, x)=\int_{0}^{t} \int_{D} W_{H}(s, y) G_{t-s}(x, y) d y d s .
$$

It belongs to $C^{1,2}\left((0, \infty) \times D \rightarrow(S)_{H}^{*}\right) \cap C\left([0, \infty) \times \bar{D} \rightarrow(S)_{H}^{*}\right)$.

b) If $H=\left(H_{0}, H_{1}, \ldots, H_{n}\right) \in\left(\frac{1}{2}, 1\right)^{n+1}$ and

$$
H_{i}>1-\frac{1}{n} \quad \text { for } \quad i=1,2, \ldots, n
$$

then $U(t, x) \in L^{2}\left(\mu_{\varphi}\right)$ for all $t \geq 0, x \in \bar{D}$.

c) In particular, for all $H \in\left(\frac{1}{2}, 1\right)^{d+1}$ we have

$$
U(t, x) \in L^{2}\left(\mu_{\varphi}\right) \quad \text { if } n \leq 2 \text {. }
$$

Remark 3.2 Note that condition (3.17) is sharp at $H_{i}=\frac{1}{2}$, in the sense that if we let $H_{i} \rightarrow \frac{1}{2}$ for $i=1, \ldots, n$ then (3.17) reduces to the condition $n=1$ which we found for the standard white noise case (3.12).

Remark 3.3 In [H1] (and more generally in [H2]) the heat equation with a fractional white noise potential is studied:

$$
\frac{\partial u}{\partial t}(t, x)=\frac{1}{2} \Delta u(t, x)+u(t, x) \diamond W_{H}(t, x) ; \quad x \in \mathbf{R}^{n}, \quad t>0 .
$$

There it is shown that if $H=\left(H_{0}, H_{1}, \ldots, H_{n}\right)$ with $H_{i} \in\left(\frac{1}{2}, 1\right)$ for $i=0,1, \ldots, n$ and

$$
H_{1}+H_{2}+\cdots+H_{n}>n-\frac{2}{2 H_{0}-1}
$$

then $u(t, x) \in L^{2}\left(\mu_{\varphi}\right)$ for all $t, x$. 


\section{The quasilinear stochastic fractional heat equation}

Let $f: \mathbf{R} \rightarrow \mathbf{R}$ be a function satisfying

$$
\begin{array}{ll}
|f(x)-f(y)| \leq L|x-y| & \text { for all } x, y \in \mathbf{R} \\
|f(x)| \leq M(1+|x|) & \text { for all } x \in \mathbf{R},
\end{array}
$$

where $L$ and $M$ are constants.

In this section we consider the following quasi-linear generalization of equation (3.1)(3.3):

$$
\begin{aligned}
& \frac{\partial U}{\partial t}(t, x)=\frac{1}{2} \Delta U(t, x)+f(U(t, x))+W_{H}(t, x) ; \quad t>0, x \in \mathbf{R}^{n} \\
& U(0, x)=U_{0}(x) ; \quad x \in \mathbf{R}^{n}
\end{aligned}
$$

where $U_{0}(x)$ is a given bounded deterministic function on $\mathbf{R}^{n}$.

We say that $U(t, x)$ is a solution of (4.3)-(4.4) if

$$
\begin{array}{rl}
\int_{\mathbf{R}^{n}} & U(t, x) \varphi(x) d x-\int_{\mathbf{R}^{n}} U_{0}(x) \varphi(x) d x \\
= & \frac{1}{2} \int_{0}^{t} \int_{\mathbf{R}^{n}} U(s, x) \Delta \varphi(x) d x d s+\int_{0}^{t} \int_{\mathbf{R}^{n}} f(U(s, x)) \varphi(x) d x d s \\
& +\int_{0}^{t} \int_{\mathbf{R}^{n}} \varphi(x) d B_{H}(s, x)
\end{array}
$$

for all $\varphi \in C_{0}^{\infty}\left(\mathbf{R}^{n}\right)$.

As in Walsh [W] we can show that $U(t, x)$ solves (4.5) if and only if it satisfies the following integral equation

$$
\begin{aligned}
U(t, x)= & \int_{\mathbf{R}^{n}} U_{0}(y) G_{t}(x, y) d y+\int_{0}^{t} \int_{\mathbf{R}^{n}} f(U(s, y)) G_{t-s}(x, y) d y d s \\
& +\int_{0}^{t} \int_{\mathbf{R}^{n}} G_{t-s}(x, y) d B_{H}(s, y),
\end{aligned}
$$

where

$$
G_{t-s}(x, y)=(2 \pi(t-s))^{-n / 2} \exp \left(-\frac{|x-y|^{2}}{2(t-s)}\right) ; \quad s<t, x \in \mathbf{R}^{n}
$$

is the Green function for the heat operator $\frac{\partial}{\partial t}-\frac{1}{2} \Delta$ in $(0, \infty) \times \mathbf{R}^{n}$. 
For the proof of our main result, we need the following two lemmas. Let $0<\alpha<1$. Define, for $u>0$,

$$
g(u, y)=\int_{\mathbf{R}}|y-z|^{-\alpha} \frac{1}{\sqrt{u}} \exp \left(-\frac{z^{2}}{2 u}\right) d z
$$

Lemma 4.1 Assume $p>\frac{1}{1-\alpha}$. Then $g(u, y) \leq C\left(1+u^{-\frac{1}{2}\left(1-\frac{1}{p}\right)}\right)$, where $C$ is a constant independent of $y$ and $u$.

Proof. In the proof, we will use $C$ to denote a generic constant independent of $y$ and $u$. First,note that

$$
g(u, y)=\int_{|z-y| \leq 1}|y-z|^{-\alpha} \frac{1}{\sqrt{u}} \exp \left(-\frac{z^{2}}{2 u}\right) d z+\int_{|z-y|>1}|y-z|^{-\alpha} \frac{1}{\sqrt{u}} \exp \left(-\frac{z^{2}}{2 u}\right) d z
$$

By Hölder inequality,

$$
\begin{aligned}
& g(u, y) \leq C\left\{1+\left[\int_{|z-y| \leq 1}|y-z|^{-\alpha \frac{p}{p-1}} d z\right]^{\frac{p-1}{p}}\left[\int_{|z-y| \leq 1} \frac{1}{u^{\frac{1}{2} p}} \exp \left(-\frac{p z^{2}}{2 u}\right) d z\right]^{\frac{1}{p}}\right\} \\
& \leq C\left(1+u^{-\frac{1}{2}\left(1-\frac{1}{p}\right)}\right)
\end{aligned}
$$

Let $F\left(y_{1}, y_{2}, \ldots, y_{n}\right)$ denote a function on $\mathbf{R}^{n}$.

Lemma 4.2 Let $h=\left(h_{1}, h_{2}, \ldots, h_{n}\right)$ with $h_{i} \geq 0,1 \leq i \leq n$. Asume that $F$ and all its partial derivatives of first order are integrable with respect to the Lebesgue measure. Then

$$
\int_{\mathbf{R}^{n}}|F(y-h)-F(y)| d y \leq \sum_{i=1}^{n}\left(\int_{\mathbf{R}^{n}}\left|\frac{\partial F}{\partial y_{i}}\left(y_{1}, y_{2}, \ldots, y_{n}\right)\right| d y\right) h_{i}
$$

Proof. Observe that

$$
\begin{gathered}
F(y-h)-F(y)=\sum_{i=1}^{n}\left(F\left(y_{1}, \ldots, y_{i-1}, y_{i}-h_{i}, y_{i+1}-h_{i+1}, \ldots, y_{n}-h_{n}\right)\right. \\
\left.-F\left(y_{1}, \ldots, y_{i-1}, y_{i}, y_{i+1}-h_{i+1}, \ldots, y_{n}-h_{n}\right)\right) \\
=\sum_{i=1}^{n} \int_{y_{i}-h_{i}}^{y_{i}}-\frac{\partial F}{\partial y_{i}}\left(y_{1}, \ldots, y_{i-1}, z, y_{i+1}-h_{i+1}, \ldots, y_{n}-h_{n}\right) d z
\end{gathered}
$$

Integrating the equation (4.11), we get 


$$
\begin{aligned}
\int_{\mathbf{R}^{n}}|F(y-h)-F(y)| d y & \sum_{i=1}^{n} \int_{\mathbf{R}^{n-1}} d y_{1} \cdots d y_{i-1} d y_{i+1} \cdots d y_{n} \\
& \cdot \int_{\mathbf{R}} d y_{i} \int_{y_{i}-h_{i}}^{y_{i}}\left|\frac{\partial F}{\partial y_{i}}\right|\left(y_{1}, \cdots, y_{i-1}, z, y_{i+1}-h_{i+1}, \cdots, y_{n}-h_{n}\right) d z \\
= & \sum_{i=1}^{n} \int_{\mathbf{R}^{n-1}} d y_{1} \cdots d y_{i-1} d y_{i+1} \cdots d y_{n} \\
& \cdot \int_{\mathbf{R}} d z\left|\frac{\partial F}{\partial y_{i}}\right|\left(y_{1}, \cdots, y_{i-1}, z, y_{i+1}-h_{i+1}, \cdots, y_{n}-h_{n}\right) \int_{z}^{z+h_{i}} d y_{i} \\
= & \sum_{i=1}^{n}\left(\int_{\mathbf{R}^{n}}\left|\frac{\partial F}{\partial y_{i}}\left(y_{1}, y_{2}, \ldots, y_{n}\right)\right| d y\right) h_{i}
\end{aligned}
$$

Our main result is the following:

Theorem 4.3 Let $H=\left(H_{0}, H_{1}, \ldots, H_{n}\right) \in\left(\frac{1}{2}, 1\right)^{n+1}$ with

$$
H_{i}>1-\frac{1}{n} \quad \text { for } i=1,2, \ldots, n \text {. }
$$

Then there exists a unique $L^{2}\left(\mu_{\varphi}\right)$-valued random field solution $U(t, x) ; t \geq 0, x \in \mathbf{R}^{n}$ of (4.3)-(4.4). Moreover, the solution has a jointly continuous version in $(t, x)$ if $H_{0}>\frac{3}{4}$.

Proof. Define

$$
V(t, x)=\int_{0}^{t} \int_{\mathbf{R}^{n}} G_{t-s}(x, y) d B_{H}(s, y) .
$$

Dividing $R$ into regions $\{z ;|z-y| \leq 1\}$ and $\{z ;|z-y|>1\}$, we see that a slight modification of the arguments in Section 4 gives that $E_{\mu_{\varphi}}\left[V^{2}(t, x)\right]<\infty$, so $V(t, x)$ exists as an ordinary random field. The existence of the solution now follows by usual Picard iteration: Define

$$
U_{0}(t, x)=U_{0}(x)
$$

and iteratively 


$$
\begin{aligned}
& U_{j+1}(t, x)=\int_{\mathbf{R}^{n}} U_{0}(y) G_{t}(x, y) d y \\
& \quad+\int_{0}^{t} \int_{\mathbf{R}^{n}} f\left(U_{j}(s, y)\right) G_{t-s}(x, y) d y d s+V(t, x) ; \quad j=0,1,2, \ldots
\end{aligned}
$$

Then by (4.2) $U_{j}(t, x) \in L_{\mu_{\varphi}}^{2}$ for all $j$. We have

$$
U_{j+1}(t, x)-U_{j}(t, x)=\int_{0}^{t} \int_{\mathbf{R}^{n}}\left[f\left(U_{j}(s, y)\right)-f\left(U_{j-1}(s, y)\right)\right] G_{t-s}(x, y) d y d s
$$

and therefore by (4.1), if $t \in[0, T]$,

$$
\begin{aligned}
& E_{\mu_{\varphi}}\left[\left|U_{j+1}(t, x)-U_{j}(t, x)\right|^{2}\right] \\
& \leq L E_{\mu_{\varphi}}\left[\left(\int_{0}^{t} \int_{\mathbf{R}^{n}}\left|U_{j}(s, y)-U_{j-1}(s, y)\right| G_{t-s}(x, y) d y d s\right)^{2}\right] \\
& \leq L\left(\int_{0}^{t} \int_{\mathbf{R}^{n}}^{t} G_{t-s}(x, y) d y d s\right) E_{\mu_{\varphi}}\left[\int_{0}^{t} \int_{\mathbf{R}^{n}}\left|U_{j}(s, y)-U_{j-1}(s, y)\right|^{2} G_{t-s}(x, y) d y d s\right] \\
& \leq C_{T} \int_{0}^{t} \sup _{y} E\left[\left|U_{j}(s, y)-U_{j-1}(s, y)\right|^{2}\right] d s \\
& \leq \ldots \leq C_{T}^{j} \int_{0}^{t} \int_{0}^{s_{1}} \ldots \int_{0}^{s_{j-1}} \sup _{y} E\left[\left|U_{1}(s, y)-U_{0}(s, y)\right|^{2}\right] d s d s_{j-1} \ldots d s_{1} \\
& \leq A_{T} C_{T}^{j} \frac{T^{j}}{(j) !} \quad \text { for some constants } A_{T}, C_{T} .
\end{aligned}
$$

It follows that the sequence $\left\{U_{j}(t, x)\right\}_{j=1}^{\infty}$ of random fields converges in $L^{2}\left(\mu_{\varphi}\right)$ to a random field $U(t, x)$. Letting $k \rightarrow \infty$ in $(4.10)$ we see that $U(t, x)$ is a solution of (4.3)-(4.4). The uniqueness follows from the Gronwall inequality. It is not difficult to see that both

$$
\int_{\mathbf{R}^{n}} U_{o}(y) G_{t}(x, y) d y \quad \text { and } \quad \int_{0}^{t} \int_{\mathbf{R}^{n}} f(U(s, y)) G_{t-s}(x, y) d y d s
$$

are jointly continuous in $(t, x)$. So to finish the proof of the theorem it suffices to prove that $V(t, x)$ has a jointly continuous version.

To this end, consider for $h \in \mathbf{R}$ 


$$
\begin{gathered}
V(t+h, x)-V(t, x)=\int_{t}^{t+h} \int_{\mathbf{R}^{d}} G_{t+h-s}(x, y) d B_{H}(s, y) \\
+\int_{0}^{t} \int_{\mathbf{R}^{n}}\left(G_{t+h-s}(x, y)-G_{t-s}(x, y)\right) d B_{H}(s, y)
\end{gathered}
$$

By the estimate in (3.15) it follows that

$$
\begin{aligned}
& E\left[\left|\int_{t}^{t+h} \int_{\mathbf{R}^{n}} G_{t+h-s}(x, y) d B_{H}(s, y)\right|^{2}\right] \leq C \int_{t}^{t+h}(u-t)^{2 H_{0}-2} d u \\
& \quad \leq C h^{2 H_{0}-1} .
\end{aligned}
$$

To estimate the second term on the right hand side of (4.15), we use (2.15) and proceed as follows:

$$
\begin{aligned}
E[\mid & \left.\left.\int_{0} \int_{\mathbf{R}^{n}}\left(G_{t+h-s}(x, y)-G_{t-s}(x, y)\right) d B_{H}(s, y)\right|^{2}\right] \\
\leq & C \iint_{\mathbf{R}} \mathcal{X}_{[0, t]}(r) \mathcal{X}_{[0, t]}(s)|r-s|^{2 H_{0}-2} \\
& \cdot\left[\int \int _ { \mathbf { R } ^ { n } \mathbf { R } ^ { n } } \left\{(t+h-r)^{-n / 2} \exp \left(-\frac{|x-z|^{2}}{2(t+h-r)}\right)\right.\right. \\
& \left.-(t-r)^{-n / 2} \exp \left(-\frac{|x-z|^{2}}{2(t-r)}\right)\right\} \\
& \cdot\left\{(t+h-s)^{-n / 2} \exp \left(-\frac{|x-y|^{2}}{2(t+h-s)}\right)\right. \\
& \left.-(t-s)^{-n / 2} \exp \left(-\frac{|x-y|^{2}}{2(t-r)}\right)\right\} \\
& \left.\cdot \prod_{i=1}^{n}\left|y_{i}-z_{i}\right|^{2 H_{i}-2} d y d z\right] d r d s \\
\leq & C \int_{\mathbf{R}} \int_{\mathbf{R}} \mathcal{X}_{[0, t]}(r) \mathcal{X}_{[0, t]}(s)|r-s|^{2 H_{0}-2} \\
& \cdot\left[\int_{\mathbf{R}^{n} \mathbf{R}^{n}}\left\{(r+h)^{-n / 2} \exp \left(-\frac{|z|^{2}}{2(r+h)}\right)-r^{-n / 2} \exp \left(-\frac{|z|^{2}}{2 r}\right)\right\}\right. \\
& \cdot\left\{(s+h)^{-n / 2} \exp \left(-\frac{|y|^{2}}{2(s+h)}\right)-s^{-n / 2} \exp \left(-\frac{|y|^{2}}{2 s}\right)\right\} \\
& \left.\cdot \prod_{i=1}^{n}\left|y_{i}-z_{i}\right|^{2 H_{i}-2} d y d z\right] d r d s
\end{aligned}
$$


From (4.17) to (4.18), we first perform the change of variables: $x-y=y^{\prime}, x-z=z^{\prime}$, $t-r=r^{\prime}, t-s=s^{\prime}$ and then we change the name of $y^{\prime}, z^{\prime}, r^{\prime}, s^{\prime}$ back to $y, z, r, s$ again for simplicitiy. (4.18) is further less than

$$
\begin{aligned}
C \int_{0}^{t} d s \int_{0}^{s} d r(s-r)^{2 H_{0}-2} \\
\cdot\left[\int_{\mathbf{R}^{n}} d y \int_{s}^{s+h}\left(-\frac{n}{2} v^{-\frac{n}{2}-1} \exp \left(-\frac{|y|^{2}}{2 v}\right)+\frac{1}{2} v^{-\frac{n}{2}-2}|y|^{2} \exp \left(-\frac{|y|^{2}}{2 v}\right)\right) d v\right. \\
\cdot\left\{(r+h)^{-n / 2} \exp \left(-\frac{|z|^{2}}{2(r+h)}\right)-r^{-n / 2} \exp \left(-\frac{|z|^{2}}{2 r}\right)\right\} \\
\left.\cdot \prod_{i=1}^{n}\left|y_{i}-z_{i}\right|^{2 H_{i}-2} d z\right] \\
\leq C \int_{0}^{t} d s \int_{0}^{s} d r(s-r)^{2 H_{0}-2} \\
\cdot\left[\int_{s}^{s+h} d v \int_{\mathbf{R}^{n}} d y\left(\frac{n}{2} v^{-\frac{n}{2}-1} \exp \left(-\frac{|y|^{2}}{2 v}\right)+\frac{1}{2} v^{-\frac{n}{2}-2}|y|^{2} \exp \left(-\frac{|y|^{2}}{2 v}\right)\right)\right. \\
\cdot \int_{\mathbf{R}^{n}}\left\{(r+h)^{-n / 2} \exp \left(-\frac{|z|^{2}}{2(r+h)}\right)+r^{-n / 2} \exp \left(-\frac{|z|^{2}}{2 r}\right)\right\} \\
\left.\cdot \prod_{i=1}^{n}\left|y_{i}-z_{i}\right|^{2 H_{i}-2} d z\right]
\end{aligned}
$$

Choose $p>1$ such that

$$
\frac{1}{2 H_{i}-1}<p<\frac{n}{n-2} \quad \text { for } i=1,2, \ldots, n \text {. }
$$

This is possible since $H_{i}>1-\frac{1}{n}$ for $i=1,2, \ldots, n$. Then

$$
\frac{2 p}{p-1}>d \quad \text { and } \quad \frac{p}{p-1}\left(2 H_{i}-2\right)>-1, \quad i=1,2, \ldots, n
$$

Now applying Lemma 4.1 repeatedly to this choice of $p$ and to $\alpha=2-2 H_{i}$, we get

$$
\begin{gathered}
(4.19) \leq C \int_{0}^{t} d s \int_{0}^{s} d r(s-r)^{2 H_{0}-2} \cdot \int_{s}^{s+h} d v \frac{1}{v}\left(1+C r^{-\frac{1}{2}\left(1-\frac{1}{p}\right)}\right)^{n} \\
\leq C \int_{0}^{t} d s \int_{0}^{s} d r \int_{s}^{s+h} d v \frac{1}{v}\left(1+r^{-\frac{n}{2}\left(1-\frac{1}{p}\right)}\right)(s-r)^{2 H_{0}-2}
\end{gathered}
$$


Choose $\beta$ such that $2-2 H_{0}<\beta<1$. It follows that (4.20) is dominated by

$$
\begin{aligned}
& C \int_{0}^{t} d s \int_{0}^{s} d r \frac{1}{s^{1-\beta}} \int_{s}^{s+h} d v \frac{1}{v^{\beta}}\left(1+r^{-\frac{n}{2}\left(1-\frac{1}{p}\right)}\right)(s-r)^{2 H_{0}-2} \\
& \leq C h^{1-\beta} \int_{0}^{t} d s \int_{0}^{s} d r \frac{1}{s^{1-\beta}} r^{-\frac{n}{2}\left(1-\frac{1}{p}\right)}(s-r)^{2 H_{0}-2} \\
& =C h^{1-\beta} \int_{0}^{t} d s \frac{1}{s^{1-\beta}}\left[\int_{0}^{\frac{s}{2}} r^{-\frac{n}{2}\left(1-\frac{1}{p}\right)}(s-r)^{2 H_{0}-2} d r+\int_{\frac{s}{2}}^{s} r^{-\frac{n}{2}\left(1-\frac{1}{p}\right)}(s-r)^{2 H_{0}-2} d r\right] \\
& \leq C h^{1-\beta} \int_{0}^{t} \frac{1}{s^{1-\beta}} s^{1-\frac{n}{2}\left(1-\frac{1}{p}\right)-\left(2-2 H_{0}\right)} d s \leq C h^{1-\beta} .
\end{aligned}
$$

On the other hand, for $k \in \mathbf{R}^{n}$ we have

$$
V(t, x+k)-V(t, x)=\int_{0}^{t} \int_{\mathbf{R}^{n}}\left(G_{t-s}(x+k, y)-G_{t-s}(x, y)\right) d B_{H}(s, y)
$$

Hence, by (4.7),

$$
\begin{aligned}
E[\mid & \left.|V(t, x+k)-V(t, x)|^{2}\right] \\
& \leq C \int_{0}^{t} \int_{0}^{t}|r-s|^{2 H_{0}-2} \\
& \cdot \iint_{\mathbf{R}^{n}}\left\{(t-r)^{-n / 2}\left(\exp \left(-\frac{|x+k-y|^{2}}{2(t-r)}\right)-\exp \left(-\frac{|x-y|^{2}}{2(t-r)}\right)\right)\right\} \\
& \cdot\left\{(t-s)^{-n / 2}\left(\exp \left(-\frac{|x+k-z|^{2}}{2(t-s)}\right)-\exp \left(-\frac{|x-z|^{2}}{2(t-s)}\right)\right)\right\} \\
& \cdot \prod_{i=1}^{n}\left|y_{i}-z_{i}\right|^{2 H_{i}-2} d y d z d r d s \\
& \leq C \int_{0}^{t} \int_{0}^{t}|r-s|^{2 H_{0}-2} \int_{\mathbf{R}^{n} \mathbf{R}^{n}}\left\{r^{-n / 2}\left(\exp \left(-\frac{|y+k|^{2}}{2 r}\right)-\exp \left(-\frac{|y|^{2}}{2 r}\right)\right)\right\} \\
& \cdot\left\{s^{-n / 2}\left(\exp \left(-\frac{|z+k|^{2}}{2 s}\right)-\exp \left(-\frac{|z|^{2}}{2 s}\right)\right)\right\} \prod_{i=1}^{n}\left|y_{i}-z_{i}\right|^{2 H_{i}-2} d y d z d r d s \\
& \leq C \int_{0}^{t} d s \int_{0}^{s} d r(s-r)^{2 H_{0}-2} \int_{\mathbf{R}^{d}} d y\left|s^{-n / 2}\left(\exp \left(-\frac{|y+k|^{2}}{2 s}\right)-\exp \left(-\frac{|y|^{2}}{2 s}\right)\right)\right| \\
& \cdot \int_{\mathbf{R}^{n}} d z\left\{r^{-n / 2}\left(\exp ^{n}\left(-\frac{|z+k|^{2}}{2 r}\right)-\exp \left(-\frac{|z|^{2}}{2 r}\right)\right)\right\} \prod_{i=1}^{n}\left|y_{i}-z_{i}\right|^{2 H_{i}-2}
\end{aligned}
$$


Applying Lemma 4.1 and Lemma 4.2 we get

$$
\begin{gathered}
(4.22) \leq C \int_{0}^{t} d s \int_{0}^{s} d r(s-r)^{2 H_{0}-2}\left(1+r^{-\frac{1}{2}\left(1-\frac{1}{p}\right)}\right)^{n} \\
\cdot \sum_{i=1}^{n}\left|k_{i}\right| \int_{\mathbf{R}^{n}} s^{-n / 2-1} \exp \left(-\frac{|y|^{2}}{2 s}\right)\left|y_{i}\right| d y \\
\leq C|k| \int_{0}^{t} d s \int_{0}^{s} d r \frac{1}{s^{\frac{1}{2}}}(s-r)^{2 H_{0}-2} r^{-\frac{n}{2}\left(1-\frac{1}{p}\right)} \\
\leq C|k| \int_{0}^{t} d s \frac{1}{s^{\frac{1}{2}}}\left[\int_{0}^{\frac{s}{2}} d r(s-r)^{2 H_{0}-2} r^{-\frac{n}{2}\left(1-\frac{1}{p}\right)}+\int_{\frac{s}{2}}^{s} d r(s-r)^{2 H_{0}-2} r^{-\frac{n}{2}\left(1-\frac{1}{p}\right)}\right] \\
\leq C|k| \int_{0}^{t} s^{2 H_{0}-\frac{n}{2}\left(1-\frac{1}{p}\right)-\frac{3}{2}} d s \leq C|k|, \quad \text { if } \quad H_{0}>\frac{3}{4} .
\end{gathered}
$$

Combining the estimates (4.16), (4.21) and (4.23) we get, for some $\beta<1$,

$$
E\left[|V(t+h, x+h)-V(t, x)|^{2}\right] \leq C\left[h^{1-\beta}+|k|\right] .
$$

Since $V(t+h, x+k)-V(t, x)$ is a Gaussian random variable with mean zero, it follows that for any $m \geq 1$

$$
\begin{aligned}
E\left[|V(t+h, x+k)-V(t, x)|^{2 m}\right] & \leq C_{m} E\left[|V(t+h, x+k)-V(t, x)|^{2}\right]^{m} \\
\leq C_{m}\left[h^{1-\beta}+|k|\right]^{m} & \leq C_{m}\left[h^{1-\beta}+|k|\right]^{m} \text { if } m \text { is big enough } .
\end{aligned}
$$

Hence by Kolmogorov's theorem we conclude that $V(t, x)$ admits a jointly continuous version.

\section{References}

[BG] F.E. Benth and H. Gjessing: A non-linear parabolic equation with noise. A reduction method. To appear in Potential Analysis.

[D] E.B. Davis: Heat Kernels and Spectral Theory. Cambridge University Press 1989.

[DHP] T. E. Duncan, Y. Hu and B. Pasik-Duncan: Stochastic calculus for fractional Brownian motion. I. Theory. To appear in SIAM Journal of Control and Optimization.

[GN] G. Gripenberg and I. Norros: On the prediction of fractional Brownian motion. J. Appl. Prob. 33 (1996), 400-410.

[H1] Y. Hu: Heat equation with fractional white noise potentials. Preprint 1998. 
[H2] Y. Hu: A class of stochastic partial differential equations driven by fractional white noise. Preprint 1999.

[HKPS] T. Hida, H.-H. Kuo, J. Potthoff and L. Streit: White Noise Analysis. Kluwer 1993.

[HØ] Y. Hu and B. Øksendal: Fractional white noise calculus and applications to finance. Preprint, Dept. of Mathematics, University of Oslo 1999.

[HØUZ] H. Holden, B. Øksendal, J. Ubøe and T. Zhang: Stochastic Partial Differential Equations. Birkhäuser 1996.

[HØS] Y. Hu, B. Øksendal and A. Sulem: Optimal portfolio in a fractional Black \& Scholes market. Preprint, Dept. of Mathematics, University of Oslo 1999.

[HØZ] Y. Hu, B. Øksendal and T. Zhang: Stochastic partial differential equations driven by multiparameter fractional white noise. Preprint, Dept. of Mathematics, University of Oslo 1999.

[J] S. Janson: Gaussian Hilbert Spaces. Cambridge University Press 1997.

[K] H.-H. Kuo: White Noise Distribution Theory. CRC Press 1996.

[MV] B.B. Mandelbrot and J.W. Van Ness: Fractional Brownian motions, fractional noises and applications. SIAM Rev. 10 (1968), 422-437.

[NVV] I. Norros, E. Valkeila and J. Virtamo: An elementary approach to a Girsanov formula and other analytic results on fractional Brownian motions. Bernoulli 5 (1999), $571-587$.

[W] J.B. Walsh: An introduction to stochastic partial differential equations. In R. Carmona, H. Kesten and J.B. Walsh (editors): École d'Été de Probabilités de Saint-Flour XIV-1984. Springer LNM 1180, pp. 265-437. 